\title{
Patient Characteristics and Pre-Existing Chronic Diseases with COVID-19 Related Outcomes: A Real- World Experience
}

\author{
Hua Zhao ${ }^{1 *}$, Bernard Fuemmeler ${ }^{2}$, Tilahun Adera ${ }^{1}$, Evan Leung ${ }^{1}$, Silviu-Alin Bacanu ${ }^{3}$ and Jie \\ Shen ${ }^{1}$ \\ ${ }^{1}$ Department of Family Medicine and Population Health, School of Medicine, Virginia Commonwealth University, Richmond, \\ United States
}

${ }^{2}$ Health Behavior and Policy, School of Medicine, Virginia Commonwealth University, Richmond, United States

${ }^{3}$ Psychiatry, School of Medicine, Virginia Commonwealth University, Richmond, United States

Corresponding author: Hua Zhao, Department of Family Medicine and Population Health, School of Medicine, Virginia Commonwealth University, Richmond, VA 23284, United States

\section{ARTICLE INFO \\ Received: 慧 October 15, 2020}

Published: 幽 October 23, 2020

Citation: Hua Zhao, Bernard Fuemmeler, Tilahun Adera, Evan Leung, Silviu-Alin Bacanu, Jie Shen. Patient Characteristics and Pre-Existing Chronic Diseases with COVID-19 Related Outcomes: A Real-World Experience. Biomed J Sci \& Tech Res 31(3)2020. BJSTR. MS.ID.005098.

\section{ABSTRACT}

Background: Significant variations in experience of the COVID-19 pandemic have been observed in the United States. However, there is currently no published study which comprehensively examines the relationship between patient characteristics and COVID-19 related health outcomes.

Methods: In this study, using aggregated real-world data extracted from TriNetx electronic medical record data from 34 hospitals around United States, we intended to fill the gap.

Results: A total of 12,555 patients aged 18-80 years old who contracted COVID-19 were identified from January 20th to April 20th, 2020. First, in the univariate analysis, we found that patients who were older (age 51-80), Black, male, and had pre-existing chronic diseases (e.g. obesity, diabetes, hypertension, and chronic kidney diseases (CKD)) had increased risk ratio (RR) of exhibiting severe outcomes, including increased C-reactive protein (CRP), decreased oxygen saturation, hospitalization, use of ventilator, and ultimately death. Next, we applied propensity score matching to match the patients based on their characteristics. We found that patients who were older, Black, male, and diagnosed with CKD had 3.69, 1.77, 1.75, and 1,61-fold increased RR of death. On the other hand, while obesity, diabetes, and hypertension had no direct relationship with death, they were associated with other severe outcomes. In further analysis by including CRP as a matching variable, death disparity by age group, race, gender, and CKD was reduced, particularly for race and CKD where a significant disparity was no longer observed.

Conclusion: In summary, our data show significant disparities in COVID-19 related outcomes by patient characteristics and further suggest that acute inflammation plays an important role in the disparity in COVID-19 death.

\section{Introduction}

The coronavirus disease 2019 (COVID-19) pandemic has maintained a massive impact on global public health and the economy. However, the health consequences differ significantly among certain groups within the population. For instance, Black families face a much higher risk of disease contraction and death than White families [1]. As of May 4, 2020, Black Americans make up 36\% of total COVID-19 cases and 52\% of total COVID-19 deaths in Chicago, despite comprising just $30 \%$ of the population. In New York City, one of the most significantly impacted cities, 31\% of COVID-19 deaths occurred in Black Americans despite Blacks 
comprising only $24 \%$ of the population [2,3]. In Michigan, while Black Americans make up only $14 \%$ of the total population, they account for $32 \%$ COVID-19 cases and $41 \%$ of COVID-19 deaths $[4,5]$.

The disparity in COVID-19 mortality among Black Americans has been attributed to the confluence of chronic diseases that already disproportionately afflict Black Americans [1]. Compared to Whites, Black Americans experience a higher prevalence of chronic diseases including asthma, hypertension, diabetes, and obesity [6]. According to the Center for Disease Control, those with underlying medical conditions are at higher risk for severe illness and death from COVID-19 [1,7]. In Chicago, 94\% of fatal COVID-19 cases have occurred in those with preexisting conditions [8]. As Black Americans disproportionately experience chronic underlying medical conditions, they are predisposed to the higher observed mortality rates from COVID-19 [5]. A recent study confirms this hypothesis using data from the 2018 Behavioral Risk Factor Surveillance System, reporting that Black Americans are more likely to have conditions associated with increased risk of illness from COVID-19 relative to white Americans [9].

However, there is no published study to thoroughly examine the relationship between chronic diseases and racial disparity in COVID-19 mortality. Additionally, no study has further assessed whether racial disparities exist in other COVID-19 related outcomes, such as disease severity, treatment, etc. Such knowledge is critically needed for not only examining the root causes of racial disparities in COVID-19 related death, but for also supporting disease surveillance and response activities. In order to thoroughly examine racial disparities in COVID-19 outcomes, we require a nationwide dataset, which has captured the events in near realtime and contains sufficient information on demographics and preexisting chronic diseases.

In this study, we plan to utilize real-world aggregated data extracted from TriNetx electronic medical record data from 34 hospitals around U.S. In the first three months of pandemic (January $20^{\text {th }}$ to April $20^{\text {th }}, 2020$ ), we intend to assess the relationship between selected patient characteristics (e.g. age, gender, race) and pre-existing chronic diseases (e.g. obesity, hypertension, diabetes, and CKD) with COVID-19 related outcomes, including death, hospitalization, use of ventilator, oxygen saturation, and CRP.

\section{Methods}

\section{Identification of COVID-19 Cases}

To identify COVID-19 cases, we searched TriNetx electronic medical record data from 34 hospitals around U.S. As the first case in U.S. was recorded on January 20th, we set the time period as the first three months of the pandemic from January $20^{\text {th }}$ to April $20^{\text {th }}$, 2020. We applied the searching criteria suggested by TriNetx. The inclusion criteria included 1) Population: 18 to 80 years, any sex; and 2) Event which occurred on or after Jan 20, 2020 characterized by one or more of the following ICD-10 diagnosis codes: B34.2 (Coronavirus infection, unspecified), B97.29 (Other coronavirus as the cause of diseases classified elsewhere), J12.81 (Pneumonia due to SARS-associated coronavirus), U07.1 (COVID-19), and U07.2 (COVID-19, virus not identified (WHO)). The exclusion criteria were the ICD-9 code 079.89 (Other specified viral infection) and Z03.818 (Encounter for observation for suspected exposure to other biological agents ruled out).

As a federated network TriNetX received a waiver from Western IRB since only aggregated counts, statistical summaries of de-identified information, but no protected health information is received, and no study specific activities are performed in retrospective analyses. TriNetX cloud-based features allow realtime access to the de-identified longitudinal clinical data along with the analytics to analyze research questions. The de-identified clinical data is aggregated directly from the electronic medical records of the participating HCOs continuously. Both the patients and HCO's as data sources stay anonymous.

\section{Outcomes}

The main outcome variable was death within a month after the diagnosis. We also included hospitalization (yes or no, from 2 weeks before to one month after diagnosis), ventilation usage (yes or no, from 2 weeks before to one month after diagnosis), oxygen saturation $(\geq 93$ or $<93$, from 5 days before to 5 days after diagnosis), and CRP $(\geq 10.0 \mathrm{mg} / \mathrm{dL}$ or $<10.0 \mathrm{mg} / \mathrm{dL}$, from 5 days before to 5 days after diagnosis). Though multiple measurements of oxygen saturation and CRP may have been recorded for the same patient, we chose to use the most recent measurement.

\section{Covariates}

Multiple covariates were included in the propensity score matching. In Table 1, age of COVID-19 diagnosis, gender, race, obesity (body mass index 30.0 or higher, yes or no, past 5 years), hypertension (I10, yes or no, past 5 years), diabetes (E11, yes or no, past 5 years), asthma (J45.9, yes or no, past 5 years), and CKD (N18.9 yes or no, past 5 years) were included as appropriate. ICD10 code was used to define the chronic diseases. In Table 2, we further included CRP the latest measurement during 5 days before to 5 days after diagnosis).

\section{Statistical Analysis}

The statistical tool embedded in TriNetx was used. Each patient characteristic variable, including age of COVID-19 diagnosis (18-51 vs 51-80 years old), gender, race (Black vs White), and pre-existing chronic diseases, including obesity (yes or no), hypertension (yes or no), diabetes (yes or no), asthma (yes or no), and CKD (yes or no), was treated as the categorical variable. The outcome variables were also treated as categorical variables, including hospitalization (yes 
or no), ventilation usage (yes or no), oxygen saturation ( $\geq 93 \mathrm{vs}<93$ ), CRP $(\geq 10.0 \mathrm{mg} / \mathrm{dL}$ vs $<10.0 \mathrm{mg} / \mathrm{dL}$ ), and death (yes or no). First, in the univariate analysis, we calculated risk ratio (RR) to assess the risk of each outcome variable between categories of each patient characteristic and pre-existing chronic disease variable. To adjust the potential co-variates, we applied propensity score matching for each comparison. The matching variables include age of COVID-19 diagnosis, gender, race, obesity, hypertension, diabetes, asthma, and CKD for Table 1, and further CRP in Table 2, as appropriate. We applied Bonferroni method to adjust for the multiple comparisons. Since the study had 74 comparisons, we set a P value of $6.76 \times 10^{-4}$ $(0.05 / 74)$ as the cutoff point for statistical significance.

Table 1: The associations between characteristics of COVID-19 patient cohort and health outcomes in matched cohorts\#.

\begin{tabular}{|c|c|c|c|c|c|c|c|c|c|c|c|}
\hline \multirow{2}{*}{$\begin{array}{l}\text { Charac- } \\
\text { teristics }\end{array}$} & \multirow[b]{2}{*}{ Number } & \multicolumn{2}{|r|}{ Death } & \multicolumn{2}{|c|}{ CRP elevation } & \multicolumn{2}{|c|}{ Oxygen saturation } & \multicolumn{2}{|c|}{ Hospitalization } & \multicolumn{2}{|c|}{ Ventilator usage } \\
\hline & & Rate & RR (95\% CI) & Rate & RR $(95 \%$ CI $)$ & Rate & $\begin{array}{l}\text { RR ( } 95 \% \\
\text { CI) }\end{array}$ & Rate & $\begin{array}{l}\text { RR ( } 95 \% \\
\text { CI) }\end{array}$ & Rate & $\begin{array}{c}\text { RR }(95 \% \\
\text { CI) }\end{array}$ \\
\hline \multicolumn{12}{|c|}{ Age group (years) } \\
\hline $18-51$ & 5,930 & $1.25 \%$ & 1 & $12.86 \%$ & 1 & $11.38 \%$ & 1 & $26.87 \%$ & 1 & $2.34 \%$ & 1 \\
\hline $51-80$ & 5,930 & $4.61 \%$ & $\begin{array}{c}3.69(2.69 \\
5.05)^{*}\end{array}$ & $25.60 \%$ & $\begin{array}{c}1.99(1.80 \\
2.20)^{*}\end{array}$ & $20.89 \%$ & $\begin{array}{c}1.82(1.63 \\
2.02)^{*}\end{array}$ & $42.18 \%$ & $\begin{array}{c}1.57(1.47 \\
1.67)^{*}\end{array}$ & $4.83 \%$ & $\begin{array}{c}2.07(1.61, \\
2.65)^{*}\end{array}$ \\
\hline \multicolumn{12}{|c|}{ By race } \\
\hline Whites & 3,549 & $3.23 \%$ & 1 & $14.42 \%$ & 1 & $17.30 \%$ & 1 & $42.31 \%$ & 1 & $4.93 \%$ & 1 \\
\hline Blacks & 3,549 & $5.70 \%$ & $\begin{array}{c}1.77(1.41 \\
2.20)^{*}\end{array}$ & $26.11 \%$ & $\begin{array}{c}1.81(1.65 \\
1.99)^{*}\end{array}$ & $14.92 \%$ & $\begin{array}{c}0.86(0.78 \\
0.96)\end{array}$ & $39.25 \%$ & $\begin{array}{c}0.93(0.88 \\
0.98)\end{array}$ & $4.38 \%$ & $\begin{array}{c}0.89(0.72, \\
1.09)\end{array}$ \\
\hline \multicolumn{12}{|c|}{ Gender } \\
\hline Women & 5,616 & $2.84 \%$ & 1 & $16.33 \%$ & 1 & $14.23 \%$ & 1 & $34.45 \%$ & 1 & $3.65 \%$ & 1 \\
\hline Men & 5,616 & $4.96 \%$ & $\begin{array}{c}1.75(1.45 \\
2.12)^{*}\end{array}$ & $23.98 \%$ & $\begin{array}{c}1.47(1.36 \\
1.58)^{*}\end{array}$ & $20.74 \%$ & $\begin{array}{c}1.46(1.34 \\
1.58)^{*}\end{array}$ & $43.11 \%$ & $\begin{array}{c}1.25(1.19 \\
1.31)^{*}\end{array}$ & $4.80 \%$ & $\begin{array}{c}1.32(1.10, \\
1.57)\end{array}$ \\
\hline \multicolumn{12}{|c|}{ Obesity } \\
\hline $\begin{array}{l}\text { Non- } \\
\text { Obese }\end{array}$ & 3,601 & $4.34 \%$ & 1 & $19.58 \%$ & 1 & $17.05 \%$ & 1 & $42.11 \%$ & 1 & $4.17 \%$ & 1 \\
\hline Obese & 3,601 & $4.86 \%$ & $\begin{array}{c}1.20(0.90 \\
1.39)\end{array}$ & $23.78 \%$ & $\begin{array}{c}1.21(1.11 \\
1.33)^{*}\end{array}$ & $22.93 \%$ & $\begin{array}{c}1.34(1.22 \\
1.48)^{*}\end{array}$ & $48.83 \%$ & $\begin{array}{c}1.16(1.10 \\
1.22)^{*}\end{array}$ & $6.64 \%$ & $\begin{array}{c}1.59(1.30 \\
1.95)^{*}\end{array}$ \\
\hline \multicolumn{12}{|c|}{ Diabetes } \\
\hline $\begin{array}{c}\text { Non- } \\
\text { Diabetes }\end{array}$ & 3,369 & $4.87 \%$ & 1 & $23.09 \%$ & 1 & $20.46 \%$ & 1 & $44.77 \%$ & 1 & $5.39 \%$ & 1 \\
\hline Diabetes & 3,369 & $5.26 \%$ & $\begin{array}{c}1.98(0.86 \\
1.36)\end{array}$ & $26.16 \%$ & $\begin{array}{c}1.13(1.04 \\
1.24)\end{array}$ & $24.55 \%$ & $\begin{array}{c}1.20(1.09 \\
1.32)^{*}\end{array}$ & $55.47 \%$ & $\begin{array}{c}1.24(1.18 \\
1.31)^{*}\end{array}$ & $6.61 \%$ & $\begin{array}{c}1.23(1.00, \\
1.51)\end{array}$ \\
\hline \multicolumn{12}{|c|}{ Hypertension } \\
\hline $\begin{array}{l}\text { Non-Hy- } \\
\text { perten- } \\
\text { sion }\end{array}$ & 5,878 & $3.80 \%$ & 1 & $21.32 \%$ & 1 & $16.62 \%$ & 1 & $30.96 \%$ & 1 & $3.15 \%$ & 1 \\
\hline $\begin{array}{l}\text { Hyper- } \\
\text { tension }\end{array}$ & 5,878 & $2.71 \%$ & $\begin{array}{c}0.71(0.54 \\
0.95)\end{array}$ & $20.58 \%$ & $\begin{array}{c}0.97(0.87 \\
1.07)\end{array}$ & $18.92 \%$ & $\begin{array}{c}1.14(1.02 \\
1.27)\end{array}$ & $45.65 \%$ & $\begin{array}{c}1.47(1.38 \\
1.58)^{*}\end{array}$ & $5.42 \%$ & $\begin{array}{c}1.72(1.34, \\
2.21)^{*}\end{array}$ \\
\hline \multicolumn{12}{|c|}{ CKD } \\
\hline Non-CKD & 1,858 & $5.97 \%$ & 1 & $23.96 \%$ & 1 & $21.72 \%$ & 1 & $49.78 \%$ & 1 & $5.85 \%$ & 1 \\
\hline CKD & 1,858 & $9.64 \%$ & $\begin{array}{c}1.61(1.28 \\
2.03)^{*}\end{array}$ & $28.61 \%$ & $\begin{array}{c}1.19(1.07 \\
1.33)\end{array}$ & $25.99 \%$ & $\begin{array}{c}1.20(1.07 \\
1.34)\end{array}$ & $68.87 \%$ & $\begin{array}{c}1.38(1.31 \\
1.46)^{*}\end{array}$ & $8.21 \%$ & $\begin{array}{c}1.40(1.10, \\
1.78)\end{array}$ \\
\hline
\end{tabular}

\#. Matching items include age of diagnosis (continuous), gender, and presence of obesity, hypertension, diabetes, and CKD, as appropriate.

*. Statistically significant after adjusting multiple comparison ( $\mathrm{P}<6.74 \times 10-4)$.

Table 2: Associations with death after matching on CRP level.

\begin{tabular}{|c|c|c|c|c|}
\hline Characteristics & Rate & Rate ratio (95\% CI) & P value & P value for RR reduction \\
\hline \multicolumn{5}{|c|}{ By age group (years old) } \\
\hline $18-51$ & $1.47 \%$ & & $<10-4$ & 0.09 \\
\hline $51-80$ & $4.11 \%$ & $2.79(2.06,3.80)$ & & \\
\hline
\end{tabular}




\begin{tabular}{|c|c|c|c|c|}
\hline \multicolumn{5}{|c|}{ By race } \\
\hline Whites & $4.02 \%$ & 1 & & \\
\hline Blacks & $5.66 \%$ & $1.31(1.04,1.60)$ & NS & 0.03 \\
\hline \multicolumn{5}{|c|}{ By gender } \\
\hline Women & $3.04 \%$ & & & \\
\hline Men & $4.63 \%$ & $1.53(1.26,1.85)$ & $<10-4$ & 0.17 \\
\hline \multicolumn{5}{|c|}{ CKD } \\
\hline Non-CKD & $6.95 \%$ & 1 & & \\
\hline CKD & $9.15 \%$ & $1.32(1.05,1.64)$ & NS & 0.11 \\
\hline
\end{tabular}

\#. Matching items include CRP level, age of diagnosis (continuous), gender, and presence of obesity, hypertension, diabetes, and CKD, as appropriate.

\section{Results}

A total of 12,555 COVID-19 cases were identified. Among them, 452 died within a month after diagnosis. First, we examined the association between patient characteristics (e.g. age group, gender, race, obesity, diabetes, hypertension, and CKD) with RR of death (Table 3). As expected, older patients (51-80 years old) were more likely to die from the disease than younger patients (18-51 years old) ( $\left.\mathrm{RR}=5.86,95 \% \mathrm{CI}: 4.69,7.67, \mathrm{P}<10^{-4}\right)$. Men had 2.01-fold higher RR of death than women (RR=2.01, 95\%CI: 1.67,
2.42, $\left.\mathrm{P}<10^{-4}\right)$. Compared to Whites, Blacks had higher RR of death $\left(\mathrm{RR}=1.91,95 \% \mathrm{CI}: 1.58,2.31, \mathrm{P}<10^{-4}\right)$. In terms of common preexisting chronic diseases, elevated RR was observed for each type. For example, compared to non-obese patients, obese patients had 1.67-fold increased RR of death from COVID-19 (RR=1.67, 95\%CI: $1.39,2.01, \mathrm{P}<10^{-4}$ ). Furthermore, patients with all four pre-existing chronic diseases had 7.23-fold increased odds of dying than those with none of the four chronic diseases (RR=7.23, 95\%CI: 5.24, 9.98, $\left.\mathrm{P}<10^{-4}\right)$.

Table 3: Characteristics of COVID-19 patient cohort and their associations with death.

\begin{tabular}{|c|c|c|c|c|c|}
\hline Characteristics & Patients & Death & Rate & Rate ratio $(95 \% \mathrm{CI})$ & $P$ value \\
\hline All patients & $12,555(100 \%)$ & 452 & $3.60 \%$ & & \\
\hline \multicolumn{6}{|c|}{ By age group (years old) } \\
\hline $18-51$ & $5,932(47.25 \%)$ & 60 & $1.01 \%$ & & \\
\hline $51-80$ & $6,623(52.75 \%)$ & 392 & $5.92 \%$ & $5.86(4.69,7.67)$ & $<10-4$ \\
\hline \multicolumn{6}{|c|}{ By race } \\
\hline Whites & $6,571(52.34 \%)$ & 200 & $3.04 \%$ & & \\
\hline Blacks & $3,562(28.37 \%)$ & 207 & $5.81 \%$ & $1.91(1.58,2.31)$ & $<10-4$ \\
\hline \multicolumn{6}{|c|}{ By gender } \\
\hline Women & $6,912(55.05 \%)$ & 172 & $2.49 \%$ & & \\
\hline Men & $5,616(44.73 \%)$ & 280 & $4.99 \%$ & $2.01(1.67,2.42)$ & $<10-4$ \\
\hline \multicolumn{6}{|c|}{ Obesity } \\
\hline Non-Obese & $8,943(71.23 \%)$ & 270 & $3.02 \%$ & & \\
\hline Obese & $3,601(28.68 \%)$ & 182 & $5.05 \%$ & $1.67(1.39,2.01)$ & $<10-4$ \\
\hline \multicolumn{6}{|c|}{ Diabetes } \\
\hline Non-Diabetes & $9,175(73.08 \%)$ & 239 & $2.61 \%$ & & \\
\hline Diabetes & $3,369(26.83 \%)$ & 213 & $6.32 \%$ & $2.43(2.03,2.91)$ & $<10-4$ \\
\hline \multicolumn{6}{|c|}{ Hypertension } \\
\hline Non-Hypertension & 6,666 (53.09\%) & 130 & $1.95 \%$ & & \\
\hline Hypertension & $5,878(46.82 \%)$ & 322 & $5.48 \%$ & $2.81(2.30,3.43)$ & $<10-4$ \\
\hline \multicolumn{6}{|c|}{ CKD } \\
\hline Non-CKD & $10,686(85.11 \%)$ & 273 & $2.56 \%$ & & \\
\hline CKD & $1,858(14.80 \%)$ & 179 & $9.63 \%$ & $3.77(3.14,4.52)$ & $<10-4$ \\
\hline
\end{tabular}

In addition to death, we also examined four other outcome variables, including elevated CRP (>=10), decreased oxygen saturation $(<93)$, hospitalization rate, and ventilator usage (Table
4). Older cases (51-80 years old) were more likely to have elevated CRP (RR=2.24, 95\%CI: 2.07, 2.43), decreased oxygen saturation $(\mathrm{RR}=2.17,95 \% \mathrm{CI}: 1.99,2.36)$, hospitalization rate $(\mathrm{RR}=2.20$, 
95\%CI: 2.09, 2.32), and ventilator usage (RR=3.19, 95\%CI: 2.61, ${ }^{4}$ ) than White cases. However, for decreased oxygen saturation, 3.90) $\left(\mathrm{P}<10^{-4}\right.$, respectively), compared to their counterparts. hospitalization rate, and ventilator usage, no statistically significant Similar significantly increased RRs were also observed for men and those with pre-existing medical conditions. For race, Black cases had higher RR of elevated CRP (RR=1.80, 95\%CI: 1.66, 1.94, $\mathrm{P}<10$ difference was observed between Black and White cases after adjusting the multiple comparison.

Table 4: Characteristics of COVID-19 patient cohort and their associations with selected outcome variables.

\begin{tabular}{|c|c|c|c|c|c|c|c|c|c|c|c|c|}
\hline \multirow{2}{*}{$\begin{array}{l}\text { Character- } \\
\text { istics }\end{array}$} & \multicolumn{3}{|c|}{ CRP elevation } & \multicolumn{3}{|c|}{ Low oxygen saturation } & \multicolumn{3}{|c|}{ Hospitalization } & \multicolumn{3}{|c|}{ Ventilator usage } \\
\hline & Rate & RR $(95 \%$ CI $)$ & $P$ value & Rate & RR $(95 \% \mathrm{CI})$ & $P$ value & Rate & RR $(95 \% \mathrm{CI})$ & $P$ value & rate & RR $(95 \% \mathrm{CI})$ & $P$ value \\
\hline \multicolumn{13}{|c|}{ By age group (years old) } \\
\hline $18-51$ & $11.39 \%$ & & & $10.28 \%$ & & & $22.79 \%$ & & & $1.89 \%$ & & \\
\hline $51-80$ & $25.55 \%$ & $\begin{array}{c}2.24(2.07 \\
2.43)\end{array}$ & $<10^{-4}$ & $22.30 \%$ & $\begin{array}{c}2.17(1.99 \\
2.36)\end{array}$ & $<10^{-4}$ & $50.20 \%$ & $\begin{array}{c}2.20(2.09 \\
2.32)\end{array}$ & $<10-4$ & $6.03 \%$ & $\begin{array}{c}3.19(2.61 \\
3.90)\end{array}$ & $<10-^{4}$ \\
\hline \multicolumn{13}{|c|}{ By race } \\
\hline Whites & $14.57 \%$ & & & $16.92 \%$ & & & $40.56 \%$ & & & $4.72 \%$ & & \\
\hline Blacks & $26.15 \%$ & $\begin{array}{c}1.80(1.66 \\
1.94)\end{array}$ & $<10^{-4}$ & $14.97 \%$ & $\begin{array}{c}0.89(0.81 \\
0.97)\end{array}$ & $\begin{array}{c}9.6 \times 10^{-} \\
3\end{array}$ & $39.28 \%$ & $\begin{array}{c}0.97(0.92 \\
1.02)\end{array}$ & 0.19 & $4.36 \%$ & $\begin{array}{c}0.92(0.77 \\
1.11)\end{array}$ & 0.4 \\
\hline \multicolumn{13}{|c|}{ By gender } \\
\hline Women & $14.86 \%$ & & & $13.04 \%$ & & & $31.92 \%$ & & & $3.36 \%$ & & \\
\hline Men & $23.97 \%$ & $\begin{array}{c}1.61(1.50 \\
1.73)\end{array}$ & $<10-^{4}$ & $21.18 \%$ & $\begin{array}{c}1.62(1.51 \\
1.75)\end{array}$ & $<10-4$ & $44.19 \%$ & $\begin{array}{c}1.38(1.33 \\
1.45)\end{array}$ & $<10-^{4}$ & $5.00 \%$ & $\begin{array}{c}1.49(1.26 \\
1.75)\end{array}$ & $<10-^{4}$ \\
\hline \multicolumn{13}{|c|}{ Obesity } \\
\hline Non-Obese & $17.06 \%$ & & & $14.32 \%$ & & & $32.42 \%$ & & & $3.04 \%$ & & \\
\hline Obese & $23.48 \%$ & $\begin{array}{c}1.38(1.28 \\
1.48)\end{array}$ & $<10-^{4}$ & $22.48 \%$ & $\begin{array}{c}1.57(1.46, \\
1.69)\end{array}$ & $<10-4$ & $49.55 \%$ & $\begin{array}{c}1.53(1.46 \\
1.60)\end{array}$ & $<10-4$ & $5.55 \%$ & $\begin{array}{c}2.19(1.86 \\
2.57)\end{array}$ & $<10-4$ \\
\hline \multicolumn{13}{|c|}{ Diabetes } \\
\hline $\begin{array}{c}\text { Non- } \\
\text { Diabetes }\end{array}$ & $15.92 \%$ & & & $13.61 \%$ & & & $29.72 \%$ & & & $2.99 \%$ & & \\
\hline Diabetes & $27.04 \%$ & $\begin{array}{c}1.70(1.58 \\
1.82)\end{array}$ & $<10-4$ & $25.01 \%$ & $\begin{array}{c}1.84(1.71 \\
1.98)\end{array}$ & $<10-4$ & $58.17 \%$ & $\begin{array}{c}1.96(1.88 \\
2.04)\end{array}$ & $<10-4$ & $7.08 \%$ & $\begin{array}{c}2.37(2.01 \\
2.79)\end{array}$ & $<10-4$ \\
\hline \multicolumn{13}{|c|}{ Hypertension } \\
\hline $\begin{array}{c}\text { Non-Hyper- } \\
\text { tension }\end{array}$ & $14.17 \%$ & & & $11.61 \%$ & & & $22.71 \%$ & & & $1.82 \%$ & & \\
\hline $\begin{array}{l}\text { Hyperten- } \\
\text { sion }\end{array}$ & $24.34 \%$ & $\begin{array}{c}1.72(1.60 \\
1.85)\end{array}$ & $<10-4$ & $22.49 \%$ & $\begin{array}{c}1.94(1.79 \\
2.10)\end{array}$ & $<10-^{4}$ & $54.15 \%$ & $\begin{array}{c}2.38(2.27 \\
2.50)\end{array}$ & $<10-4$ & $6.68 \%$ & $\begin{array}{c}3.68(3.30 \\
4.46)\end{array}$ & $<10-4$ \\
\hline \multicolumn{13}{|c|}{ CKD } \\
\hline Non-CKD & $17.22 \%$ & & & $15.05 \%$ & & & $31.96 \%$ & & & $3.42 \%$ & & \\
\hline CKD & $28.98 \%$ & $\begin{array}{c}1.68(1.55 \\
1.82)\end{array}$ & $<10-4$ & $26.37 \%$ & $\begin{array}{c}1.75(1.61 \\
1.91)\end{array}$ & $<10-^{4}$ & $69.59 \%$ & $\begin{array}{c}2.18(2.09 \\
2.27)\end{array}$ & $<10-^{4}$ & $8.09 \%$ & $\begin{array}{c}2.37(1.98 \\
2.83)\end{array}$ & $<10-4$ \\
\hline
\end{tabular}

Given that patient characteristics were associated with COVID-19 outcome variables, we attempted to adjust them using 1 to 1 propensity score matching. The matching criteria included age of diagnosis, gender, presence of obesity, diabetes, hypertension, and CKD as appropriate (Table 1). After matching, older cases had statistically significantly higher $\mathrm{RR}$ of death (RR=3.69, 95\%CI: $2.69,5.05)$, elevated CRP (RR=1.99, 95\%CI: 1.80, 2.20), decreased oxygen saturation (RR=1.82, 95\%CI: 1.63, 2.02), hospitalization $(\mathrm{RR}=1.54,95 \% \mathrm{CI}: 1.47,1.67)$, and ventilator usage $(\mathrm{RR}=2.07$, 95\%CI: 1.61, 2.65, $\left.\mathrm{P}<10^{-4}\right)$ than younger individuals $\left(\mathrm{P}<10^{-4}\right.$, respectively). Similar significantly higher RRs were also observed for men compared to women, except in ventilator usage. Racial difference was only significant for death (RR=1.77 95\%CI: 1.41,
2.20) and elevated CRP (RR=1.81, 95\%CI: 1.65, 1.99). Obesity was associated with increased risk ratio of elevated CRP, decreased oxygen saturation, hospitalization, and ventilator usage, but not for death.

Additionally, significant associations were observed in diabetes with decreased oxygen saturation and hospitalization, hypertension with hospitalization and ventilator usage, and chronic kidney disease with death, elevated CRP, and hospitalization. Finally, we attempted to assess whether elevated CRP affected the association between age group, race, gender, chronic kidney disease with death. We utilized propensity score matching and added CRP level in the matching criteria in addition to the variables mentioned above (Table 2). We found that risk ratio was decreased for all four tested 
variables. Particularly, the associations between race $(\mathrm{RR}=1.31$, 95\%CI: $1.04,1.60)$ and CKD (RR=1.32, 95\%CI: 1.05, 1.64) with death were no longer statistically significantly after adjusting the multiple comparison. We then compared RR before and after the CRP matching, and found that the reduction was statistically significant for race $(\mathrm{P}=0.03)$.

\section{Discussion}

This is the first study which incorporates nationwide realworld data to assess patient characteristics and pre-existing chronic diseases among COVID-19 patients and their associations with COVID-19 related health outcomes. The results from our study further confirm that those who were older, Black, and had chronic diseases were at increased risk of dying or experiencing severe disease outcomes, such as increased CRP, decreased oxygen saturation, hospitalization, and ventilator usage. In addition, we found that men had a greater likelihood of experiencing severe disease outcomes compared to women. We also found that a certain proportion of disparity in mortality by age group, race, gender, and chronic kidney disease could be attributed to the difference in CRP level, an indicator of acute inflammation.

The gender difference in COVID-19 related health outcomes were particularly interesting. Similar results were also reported previously [10,11]. As we did not have data on cigarette smoking history and other lifestyle factors, we could not assess whether the observed gender difference is simply a reflection of the difference in lifestyle factors. Given that COVID-19 is a respiratory disease, it is possible that men consume more cigarettes and thereby have worse lung function than women, leading to worse COVID-19 related health outcomes, including death, increased CRP, decreased oxygen saturation, and hospitalization.

Compared to non-obese patients, it has been reported previously that obese patients are more likely to die from COVID-19 contraction [12]. However, we only observed a significant association in unmatched samples, but not in the matched samples, suggesting that obesity may not be directly related to COVID-19 death. Intriguingly, although obesity was not directly associated COVID-19 death, obese patients were more likely to have other COVID-19 related outcomes, including increased CRP, decreased oxygen saturation, increased hospitalization, and ventilator usage, all of which may eventually contribute to death. Thus, our data support an indirect association between obesity and COVID-19 related death.

The COVID-19 virus is a novel virus and is therefore able to circumvent recognition by the host. It is hypothesized that the innate immune response of the host is likely to be fully activated and release various types of cytokines and other factors (REF). However, when the immune system becomes unchecked, it will begin to destroy its own tissue and consequently lead to unfavorable health consequences and death [13]. CRP is one of main cytokines released during this surge. In this study, we found that patients who were older, Black, male, and obese had increased risk of increased CRP around COVID-19 diagnosis, suggesting the occurrence of acute inflammation. We also found that increased CRP was not only associated with an increased risk of death, but may also modify the significant associations between age, gender, race, and CKD with death.

The relationship is particularly interesting for race. After adjusting CRP, the significant association between race and death disappeared. Our data may suggest that Blacks have higher innate immune response to COVID-19 contraction and may be likely to release more cytokines (e.g. CRP, IL-6, interferon) than Whites. Such elevated innate immune response may lead to higher mortality than Whites. Though we don't have adequate statistical tools available to clarify whether elevated CRP may mediate the relationship between race and COVID-19 death, our observation is actually supported by lines of evidences. Racial difference in stress and immune function has been reported previously [14]. Racism and other social-structural factors (e.g. social economic status, housing, and neighborhood environment) may heighten the immune reactivity of Black population. The racial difference could also be due to the genetic difference. In 2016, two studies published in Cell $[15,16]$ showed that upon exposure to pathogens (e.g. bacteria and virus), the transcriptional response was higher in monocytes and macrophages obtained from Africans than in those from Europeans, indicating higher innate immune response in Africans. These studies suggested that the increased innate immune response is mainly due to differences at the genetic level directing the immune systems of people with European and African ancestry.

Interestingly, recent studies have suggested the possible role of Vitamin D in suppressing cytokine storm and associated mortality in COVID-19 Patients [17,18]. Vitamin D can suppress cytokine production by simultaneously boosting the innate immune system (thus reducing the viral load) and decreasing the overactivation of the adaptive immune system to immediately respond to the viral load [19]. Some researchers have suggested the potential role of Vitamin D in suppressing cytokine storm during the 1918-1919 viral influenza pandemic [20]. Moreover, the role of Vitamin D in enhancing immune response in flu and previous coronaviruses has been suggested $[19,21]$. Blacks are known to have lower levels of circulating vitamin D than their White counterparts [22]. Such racial difference in vitamin D levels may lead to less suppression of cytokine storm and consequently more death among Black COVID-19 patients than White COVID-19 patients. In this study, only $7 \%$ of COVID-19 patients had 25-hydroxy-vitamin D [25(OH) D] measurement. So, we don't have the power to assess the role of vitamin D in racial disparity of COVID-19 related death. 
Our study maintains several strengths and limitations. For one, the sample size is large with over 12,500 cases. As real-world samples are extracted from a nationwide network of 34 hospitals, they may have less selection bias and thereby provide better representation and generalization. A second major strength is the utilization of real-time data. The first reported case in the United States occurred on January $20^{\text {th }}, 2020$. In just three months, we already have aggregated data on over 12,500 cases. The major weakness of this study is the lack of individual data, as only aggregated data were available. Thus, the scope of data is limited. Additionally, data on healthy behavior are missing. Embedded statistical tool in TriNetx is also limited, inhibiting the performance of advanced analysis. Nevertheless, our study is the first to use real-world data to assess the relationship between personal characteristics and pre-existing chronic diseases with COVID-19 related health outcomes. The results from this study will help us better understand the disparity of COVID-19 related health outcomes among the population and identify means to reduce the disparities and improve the outcomes.

\section{Acknowledgement}

The project described was supported by CTSA award No. UL1TR002649 from the National Center for Advancing Translational Sciences. Its contents are solely the responsibility of the authors and do not necessarily represent official views of the National Center for Advancing Translational Sciences or the National Institutes of Health.

\section{References}

1. Yancy CW (2020) COVID-19 and African Americans. JAMA 323(19): 1891-1892.

2. Rafi Kabarriti, N Patrik Brodin, Maxim I Maron, Chandan Guha, Shalom Kalnicki, et al. (2020) Association of Race and Ethnicity with Comorbidities and Survival Among Patients With COVID-19 at an Urban Medical Center in New York. JAMA 3(9): e2019795.

3. (2019) Population QuickFacts, New York city, New York. United States Census.

4. (2020) Coronavirus Michigan Data.

5. (2019) Population QuickFacts, Michigan. United States Census.

6. Cunningham TJ, Croft JB, Liu Y, Lu H, Eke PI, et al. (2017) Vital Signs: Racial Disparities in Age-Specific Mortality Among Blacks or African Americans - United States, 1999-2015. MMWR Morb Mortal Wkly Rep 66(17): 444-56.

7. Williams DR, Collins C (2001) Racial residential segregation: a fundamental cause of racial disparities in health. Public Health Rep 116(5): 404-16.
8. (2020) COVID-19 Morbidity and Mortality by Geography.

9. Raifman M RaJ (2020) Disparities in the Population at Risk of Severe Illness From COVID-19 by Race/Ethnicity and Income. Am J Prev Med $59(1)$.

10. Richardson S, Hirsch JS, Narasimhan M, Crawford JM, Mcginn T, et al. (2020) Presenting Characteristics, Comorbidities, and Outcomes Among 5700 Patients Hospitalized With COVID-19 in the New York City Area. JAMA 323(20).

11.(2020) Novel Coronavirus Pneumonia Emergency Response Epidemiology $\mathrm{T} 2020$. The epidemiological characteristics of an outbreak of 2019 novel coronavirus diseases (COVID-19) in China. 41(2): 145-151.

12. Matthew J Belanger, Angeliki M Angelidi, Maria Dalamaga, James R Sowers, Christos S Mantzoros (2020) Covid-19 and Disparities in Nutrition and Obesity. New England Journal of Medicine 383: e69.

13. Merad M, Martin JC (2020) Pathological inflammation in patients with COVID-19: a key role for monocytes and macrophages. Nat Rev Immunol 20: 355-362.

14. Dowd JB, Palermo T, Chyu L, Adam E, McDade TW (2014) Race/ethnic and socioeconomic differences in stress and immune function in The National Longitudinal Study of Adolescent Health. Soc Sci Med 115: 4955.

15. Quach H, Rotival M, Pothlichet J, Eddie Loh YH, Dannemann M, et al. (2016) Genetic Adaptation and Neandertal Admixture Shaped the Immune System of Human Populations. Cell 167(3): 643-56.

16. Nedelec Y, Sanz J, Baharian G, Zachary A Szpiech, Alain Pacis, et al. (2016) Genetic Ancestry and Natural Selection Drive Population Differences in Immune Responses to Pathogens. Cell 167(3): 657-669.E21.

17. Paul E Marik, P Kory, Varon V (2020) Does vitamin D status impact mortality from SARS-CoV-2 infection? Med Drug Discov 6: 100041.

18. Biesalski HK (2020) Vitamin D deficiency and co-morbidities in COVID-19 patients - A fatal relationship? NFS Journal 20: 10-21.

19. Azrielant S, Shoenfeld Y (2017) Vitamin D and the Immune System. Isr Med Assoc J 19(8): 510-511.

20. Grant WB, Giovannucci E (2009) The possible roles of solar ultraviolet-B radiation and vitamin $\mathrm{D}$ in reducing case-fatality rates from the 1918 1919 influenza pandemic in the United States. Dermatoendocrinol 1(4): 215-219.

21. Goncalves-Mendes N, Talvas J, Duale C, Guttmann A, Corbin V, et al. (2019) Impact of Vitamin D Supplementation on Influenza Vaccine Response and Immune Functions in Deficient Elderly Persons: A Randomized Placebo-Controlled Trial. Front Immunol 10: 65.

22. Alzaman NS, Dawson-Hughes B, Nelson J, D’Alessio D, Pittas AG (2016) Vitamin D status of black and white Americans and changes in vitamin D metabolites after varied doses of vitamin D supplementation. Am J Clin Nutr 104(1): 205-214. 
ISSN: 2574-1241

DOI: 10.26717/BJSTR.2020.31.005098

Hua Zhao. Biomed J Sci \& Tech Res

(c) (P) This work is licensed under Creative BY Commons Attribution 4.0 License

Submission Link: https://biomedres.us/submit-manuscript.php

BIOMEDICAL
RESEARCHES $\quad \begin{aligned} & \text { Assets of Publishing with us } \\ & \text { - Global archiving of articles }\end{aligned}$

